\title{
Squamous Cell Carcinoma of the Vulva: Prognostic Factors Influencing Survival
}

\author{
Michael P. Hopkins, M.D., ${ }^{1}$ Gary C. Reid, M.D., Ivanna Vettrano, M.D., and George W. Morley, M.D. \\ Department of Obstetrics and Gynecology, University of Michigan Medical Center, Ann Arbor, Michigan
}

Received December 26, 1990

One hundred seventy-two cases of patients with squamous cell cancer of the vulva treated at the University of Michigan Medical Center from 1975 to 1988 are reported. The mean age was 66 years with a range of 21 to 101 years. The distribution by stage included Stage I, 65; Stage II, 44; Stage III, 50; and Stage IV, 13 patients. Groin node dissections performed on 145 patients showed negative nodes, $58 \%$; unilateral positive nodes, $28 \%$; and bilateral positive nodes, $14 \%$. The distribution of patients with positive nodes was influenced by stage: Stage I, 14\%; Stage II, 23\%; Stage III, $72 \%$; Stage IV, $92 \%$. The overall cumulative 5year survival was $71 \%$ and this was significantly influenced by stage of disease: Stage 1, 94\%; Stage II, 91\%; Stage III, 36\%; Stage IV, 26\%. Stages I/II and III/IV were combined for analysis. In Stages I/II, survival was significantly influenced by tumor grade while size, patient age, and lymph node status did not influence survival. In Stage III/IV, survival was significantly influenced by tumor size, node status, and number of positive nodes while grade, patient age, and tumor location did not influence survival. Squamous cell cancer of the vulva is effectively treated with radical surgery but advanced-stage disease with regional metastases significantly alters survival. (c) 1991 Academic Press, Inc.

\section{INTRODUCTION}

Carcinoma of the vulva tends to be slow growing and, since it is an exterior disease on the vulva, should be amenable to treatment. Early in this century the necessity for radical resection of this disease was established $[1,2]$. An earlier report from our institution demonstrated that when radical resection is undertaken, the prognosis for this malignancy can be favorable [3]. This report was undertaken to analyze and update our experience with squamous cell carcinoma of the vulva.

\footnotetext{
1 To whom reprint requests should be addressed at Northeastern Ohio Universities College of Medicine, 400 Wabash Ave.-A.C.C., Akron, OH 44307.
}

\section{MATERIAL AND METHODS}

A review of the clinical records, pathology reports, and tumor conference notes for patients diagnosed with carcinoma of the vulva for the time period 1975-1988 was undertaken. All pathology was reviewed at the Gynecologic Tumor Conference, where recommendations for therapy were made. The original hematoxylin and eosin pathology slides were reviewed for patients with superficial lesions. In general well-differentiated tumors had little nuclear pleomorphism, cytologic atypia was not pronounced, there was a more normal nuclear to cytoplasmic ratio, and keratin production was abundant. Poorly differentiated tumors had smaller cells, frequent mitosis, pronounced nuclear pleomorphism, and hyperchromatic nuclei, and keratin formation was absent. Moderately differentiated tumors were intermediate in characteristics. Information for the clinical presentation, medical history, height, weight, and treatment were recorded. At the time of diagnosis all patients were staged according to the International Federation of Gynecology and Obstetrics (FIGO) guidelines utilizing the $\mathrm{T}-\mathrm{N}-\mathrm{M}$ system. The new FIGO guidelines were not applied in a retrospective fashion. Survival was plotted with the life table method described by Kaplan and Meier [4]. The difference in survival for various parameters was tested for significance by the generalized Wilcoxon (Breslow) methods [5].

\section{RESULTS}

During the time period 1975-1988, 204 patients with carcinoma of the vulva were treated at the University of Michigan Medical Center. During this time period, 172 (85\%) patients with squamous cell carcinoma of the vulva were evaluated. The other cell types included adenocarcinoma (9), melanoma (8), Paget's disease (7), sarcoma (5), and basal cell (3). The distribution by stage for pa- 
tients with squamous cell cancer included Stage I, 65; Stage II, 44; Stage III, 50; and Stage IV, 13 patients. The mean age was 66 years with a range of 21 to 101 years. The mean age of the patients according to the stage of disease included Stage I, 60.6 years; Stage II, 68.2 years; Stage III, 71.5 years; and Stage IV, 64.2 years. The clinical characteristics included Caucasian race, 169/172 (98\%); obesity, 64/170 (38\%); smoking, 44/166 (27\%); diabetes, 21/134 (16\%); and hypertension, 54/134 (40\%). The malignancy was located on the right side in 49 patients $(30 \%)$, was on left side in 42 patients $(26 \%)$, and was bilateral in 71 patients $(44 \%)$. The location of the lesion included labium minus, 30 (18\%); labium majus, $49(30 \%)$; perineum, $28(17 \%)$; clitoris, $44(27 \%)$; and entire vulva, $14(8 \%)$. Some type of vulvectomy was performed on 169 patients. The following surgical approaches were used: en bloc butterfly, 94 (55\%); three separate incisions, 42 (25\%); radical vulvectomy only, 15 (9\%) (5 for palliation); and radical hemivulvectomy, $18(11 \%)$. Nine patients $(5 \%)$ who presented with advanced lesions had palliative therapy which included vulvectomy (six), radiation therapy (two), and pain management (one).

Groin node dissection was performed on 145 patients and $84(58 \%)$ had negative nodes, $41(28 \%)$ had positive unilateral nodes, and $20(14 \%)$ had bilateral groin nodes involved with metastatic disease. A unilateral groin node dissection was performed on 11 patients with Stage I or Stage II disease. The distribution of positive lymph nodes by stage included Stage I, 14\% (all unilateral); Stage II, 23\% (all unilateral); Stage III, 72\%; and Stage IV, $92 \%$. Thirty-eight patients underwent pelvic node dissection. Twenty-five paticnts had ncgative pelvic lymph nodes and thirteen patients had positive pelvic lymph nodes. All paticnts with positive pelvic nodes had positive groin nodes. The distribution of positive pelvic nodes by stage included Stage I, 0/4; Stage II, 0/8; Stage III, 7/18 $(39 \%)$; and Stage IV, 6/8 (75\%).

All 172 patients were available for follow-up except for 1 patient, who was lost to follow-up immediately after treatment. In this group of 171 patients, the 2-year cumulative survival was $75 \%$, and the 5 -year cumulative survival was $71 \%$. The cumulative 5 -year survival was significantly influenced by the stage of disease: Stage I, 94\%; Stage II, 91\%; Stage III, 36\%; Stage IV, 26\% (Fig. 1). There was no significant difference in the survival between Stages I and II. These two groups had a combined cumulative 5-year survival of $93 \%$. There was no significant difference in survival between Stage III and IV disease. These two groups had a combined cumulative 5 -year survival of $33 \%$. The differences in stage of disease overwhelmingly influenced survival and, for this reason, Stages I/II and Stages III/IV were combined for further analysis.

In Stage I/II disease, all patients had negative surgical

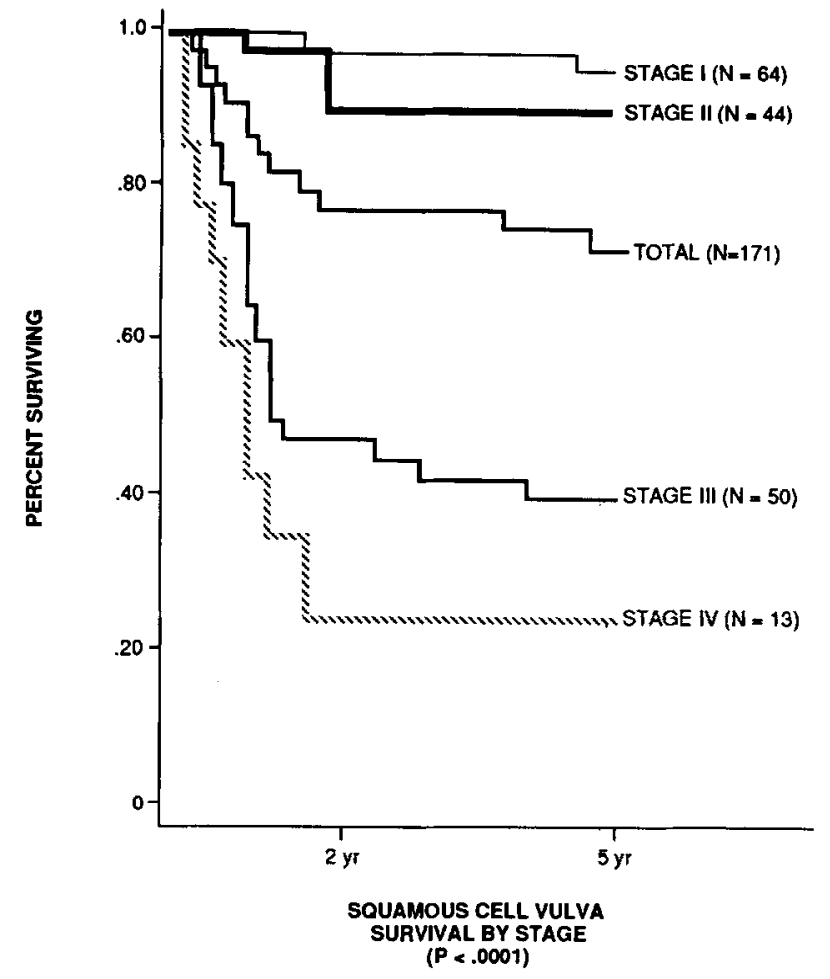

FIG. 1. Survival for patients squamous cell cancer of the vulva by stage of disease.

margins and the factors that did not significantly influence survival included the size of the tumor, the age of the patient, the lymph node status, the location of the tumor, and the type of surgery. Patients with a poorly differentiated tumor had a significantly worse survival than those with a well-differentiated or moderately differentiated tumor (Fig. 2). Twenty-nine patients with Stage I disease had a superficially invasive lesion to a depth of 2 $\mathrm{mm}$ or less. Twenty-four patients had a depth of invasion less than $1 \mathrm{~mm}$, and five patients had 1- to $2-\mathrm{mm}$ penetration. There were no deaths among these patients with superficial lesions. The following treatments were employed for these superficial lesions without a vulvar recurrence: butterfly en bloc radical vulvectomy, 6; three separate incision radical vulvectomy, 5; radical vulvectomy, 6. The 11 patients who had groin node dissection all had negative lymph nodes. A hemivulvectomy, in a wide excisional fashion, was performed for the remaining 12 patients. Two of these twelve patients developed a recurrence. One patient had $1 \mathrm{~mm}$ of invasion. Two years later she had $2 \mathrm{~mm}$ of invasion and a radical vulvectomy was performed. One patient had less than $1 \mathrm{~mm}$ of invasion and 6 years later underwent wide local excision for carcinoma in situ.

Patients with Stage III/IV disease were then analyzed for factors related to survival. The grade of tumor, age 


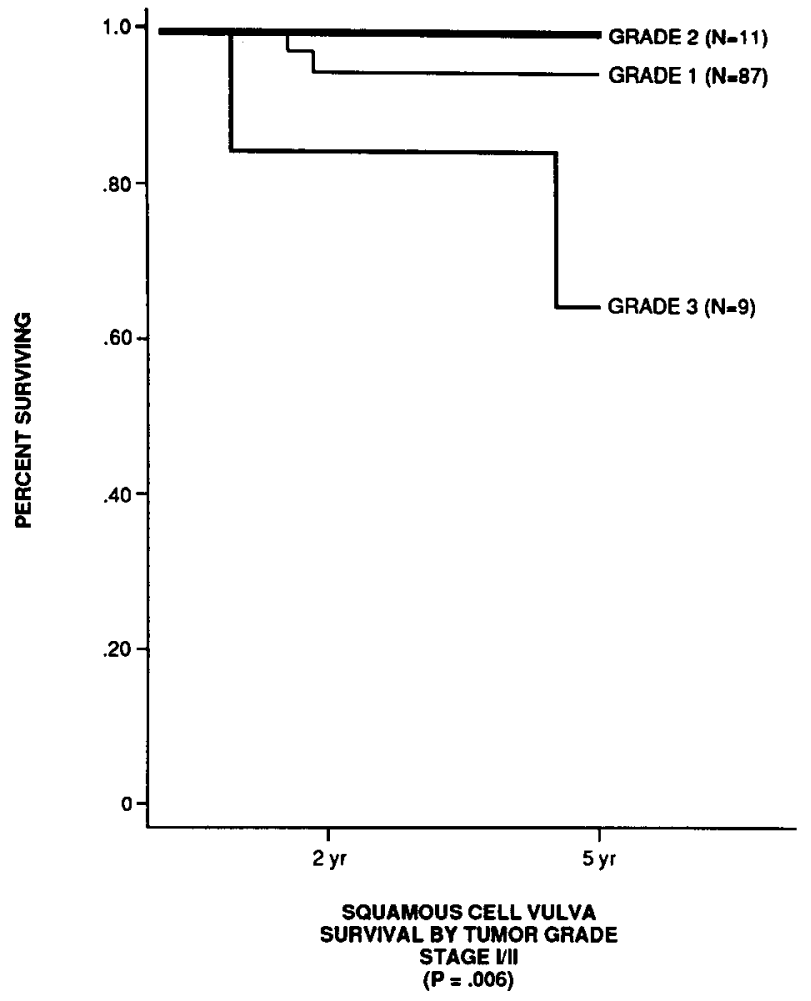

FIG. 2. Survival for patients with Stage I/II disease by tumor differentiation.

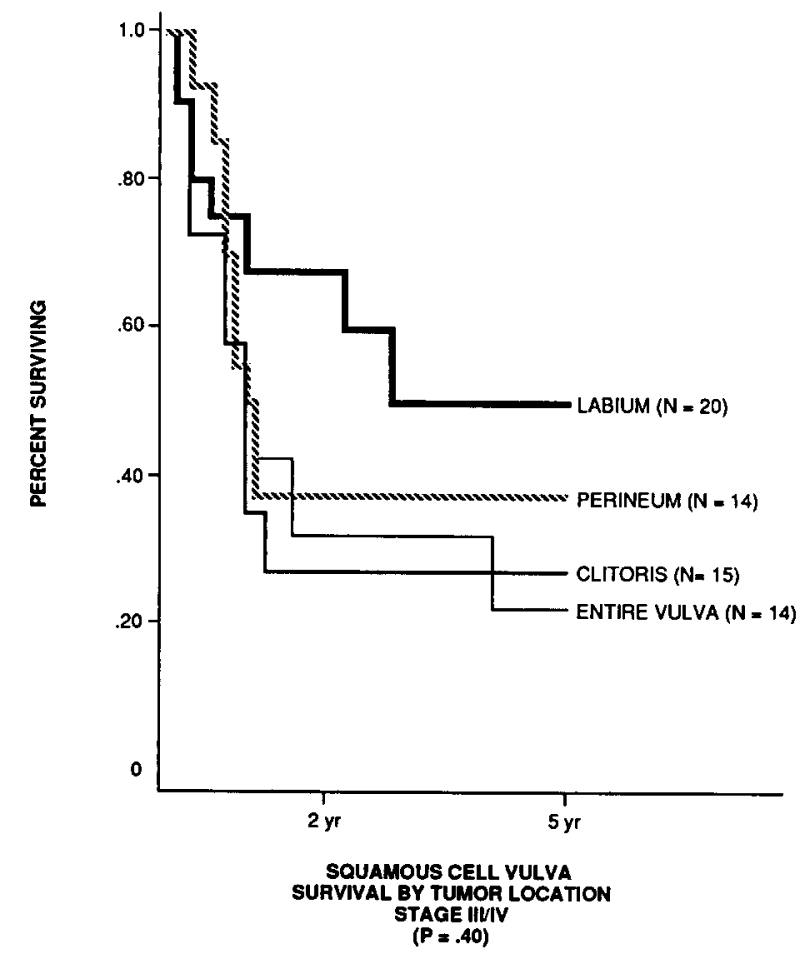

FIG. 3. Survival for patients with Stage III/IV disease by tumor location. of the patient, and tumor location (Fig. 3) did not significantly influence survival. Factors found to significantly influence survival included the size of the lesion (Fig. 4), the lymph node status (Fig. 5), and the pelvic lymph node status (Fig. 6). In Stage III/IV disease, the presence of unilateral positive groin nodes decreased survival to $30 \%$ while the presence of bilateral groin lymph nodes decreased survival to $7 \%$. The presence of positive pelvic nodes decreased survival to $8 \%$. No patient with negative groin nodes had positive pelvic lymph nodes. The total number of positive lymph nodes significantly influenced survival (Fig. 7).

Postoperative complications included 1 postoperative death from gram negative sepsis, which occurred in 1976. A total of 86 patients $(50 \%)$ experienced some type of wound complication. These complications included wound infection, 28; wound cellulitis, 25; lymphocyst, 33; and groin wound breakdown, 82. Twenty-five patients $(17 \%)$ developed lymphedema, but none of these patients experienced severe debilitating lymphedema. Ten patients $(7 \%)$ experienced a problem with chronic cellulitis, thought to be related to the groin lymph node dissection.

Thirty-two patients were available for evaluation of recurrent disease. Twenty patients had local/regional recurrence and twelve had distant metastases. All 12 patients who developed distant metastatic diseasc had original Stage III/IV disease.

\section{DISCUSSION}

Squamous cell carcinoma of the vulva is a disease that tends to remain localized for extended periods of time. In this report there is a difference in mean age between Stage I and III of 11 years. This could be explained by a progression interval of approximately one decade from early to advanced disease. Alternatively it is possible that the elderly patient presents at a later date. The 2-year survival of $75 \%$ and the 5 -year survival of $71 \%$ reported here attest to the fact that excellent survival can be obtained with the primary surgical approach. Thus surgery should remain the primary mode of therapy for this disease and the present survival results are similar to the $73.9 \%$ previously reported from our institution for the 40-year time period 1935-1975 [3].

The most significant factor for predicting survival in this disease remains the stage of the disease. Patients diagnosed early, prior to the presence of clinically positive lymph nodes, have a prognosis significantly improved over that of patients with advanced-stage disease, which often involves regional lymph nodes. The stage of disease significantly influences survival, as has been confirmed by other authors. In the present study the survival is similar stage for stage to that previously reported by both our institution and other institutions $[3,6]$. Pao et al. reported 


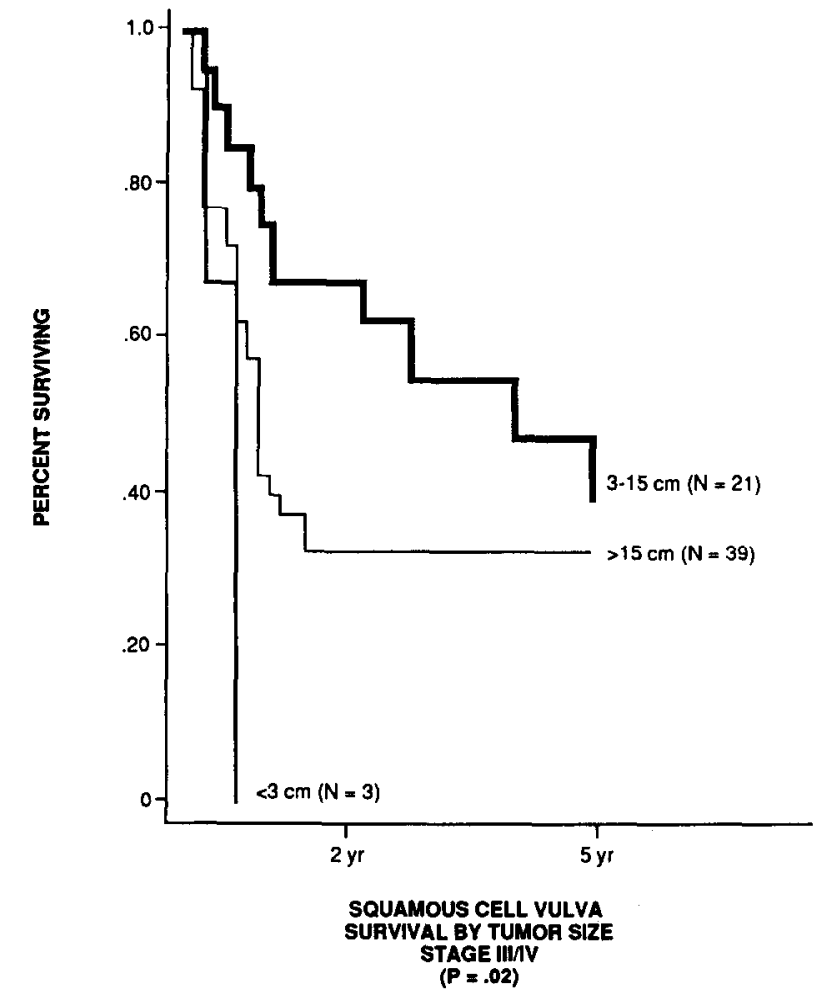

FIG. 4. Survival for patients with Stage III/IV disease according to the tumor size.

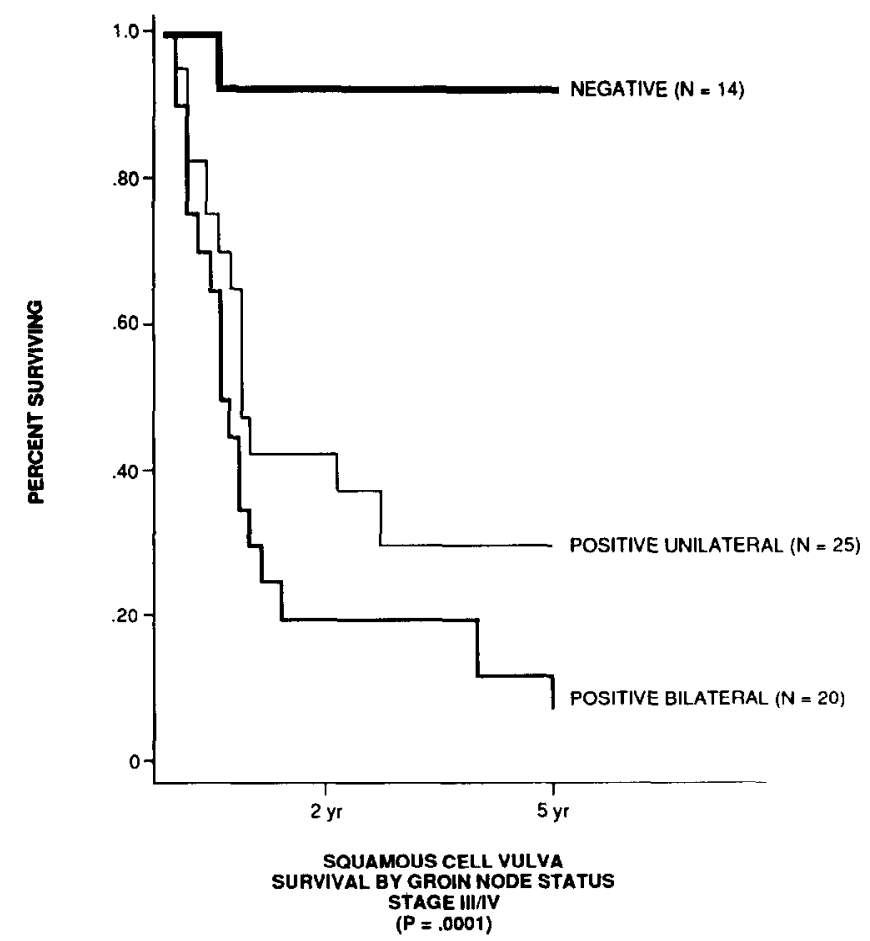

FIG. 5. Survival for patients with Stage III/IV disease according to the groin lymph node status.

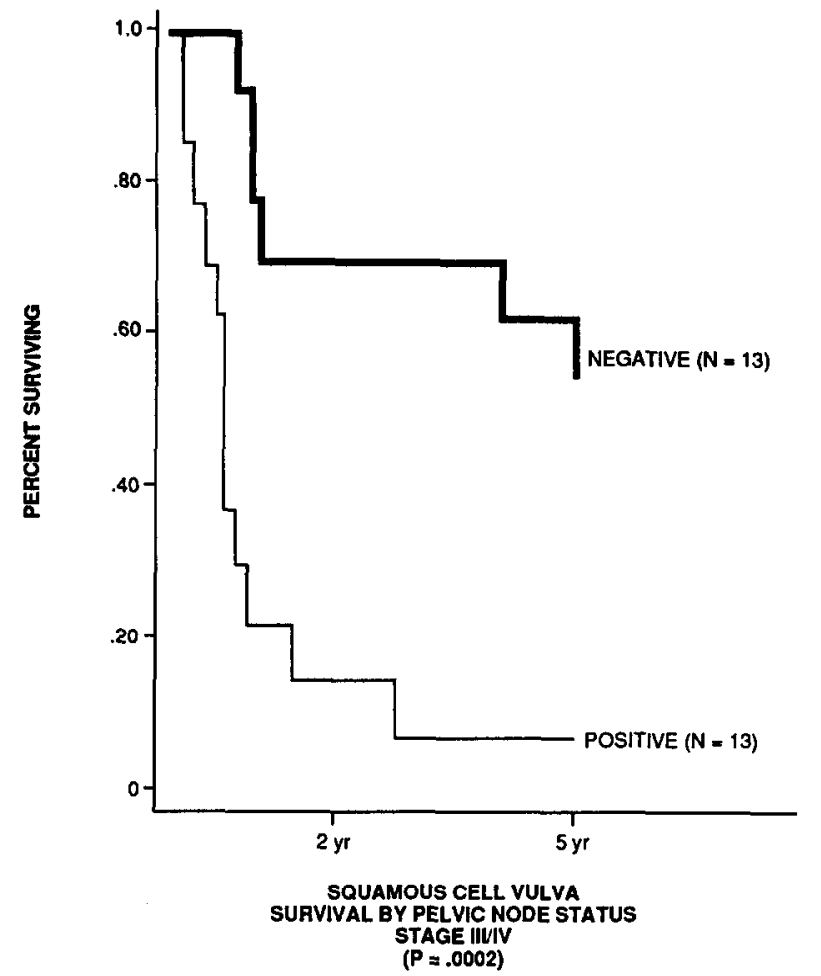

FIG. 6. Survival for patients with Stage III/IV disease according to the pelvic lymph node status.

a decrease in survival to $50 \%$ for Stage III disease and to $0 \%$ for Stage IV disease [7]. Shimm et al., like us, reported that survival was significantly related to the stage of disease, the size of the tumor, and the lymph node status [8]. There has been a marked decrease in the number of patients presenting with advanced lesions where only palliation is offered. In the previous time period, 63 patients $(21 \%)$ presented with disease amenable only to palliation while in this reported time span only $9 / 172$ (5\%) from our institution were considered for palliation [3].

As in other reports, our study found the presence of unilateral or bilateral node metastases and pelvic node involvement to be a significant predictor of survival [79]. Additionally the total number of involved nodes predicts extremely high risk groups. Unlike advanced-stage disease, however, node metastases in Stage I/II do not carry an unfavorable prognosis. This was our experience in the years prior to 1975 and it is demonstrated in this report. Hoffman et al., in analyzing the microscopic features of involved lymph nodes, reported that small foci in nodes did not alter prognosis while nodes largely replaced by tumor did decrease survival [10]. The patients with Stage I/II disease had clinically nonsuspicious nodes consistent with microscopic disease while patients with Stages III/IV often had large palpable suspicious nodes. 


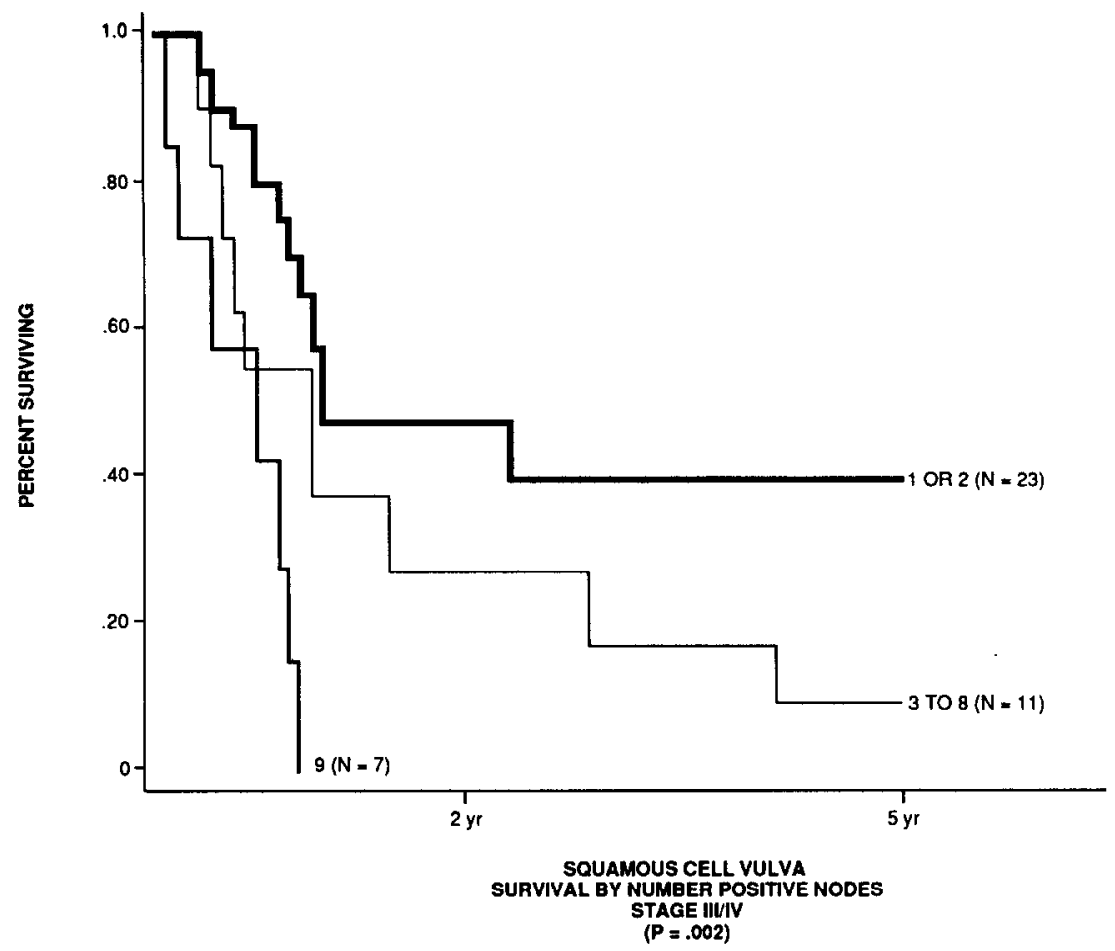

FIG. 7. Survival for patients with Stage III/IV disease according to the number of positive lymph nodes.

This report confirms that squamous cell carcinoma of the vulva has an overall favorable prognosis. The greatest challenge however remains earlier diagnosis prior to the presence of advanced local disease or metastatic spread to lymph nodes.

\section{REFERENCES}

1. Taussig, F. J. Cancer of the vulva, Am. J. Obstet. Gynecol. 40, 764-767 (1940).

2. Way, S. Carcinoma of the vulva, Am. J. Obstet. Gynecol. 79, 692697 (1960)

3. Morley, G. W. Infiltrative carcinoma of the vulva: Results of surgical treatment, Am. J. Obstet. Gynecol. 124, 874-884 (1976).

4. Kaplan, E. L., and Meier, P. Non parametric estimation from incomplete observations, J. Am. Statist. Assoc. 53, 457-481 (1958).

5. Breslow, N. A generalized Kruskal-Wallis test for comparing $K$ samples subject to unequal patterns of censorship, Biometrika 57, 179-594 (1970).

6. Podratz, K. C., Symmonds, R. E., Taylor, W. F., and Williams, T. J. Carcinoma of the vulva: Analysis of treatment and survival, Obstet. Gynecol. 61, 63-74 (1983).

7. Pao, W. M., Perez, C. A., Kuske, R. R., Sommers, G. M., Camel, H. M., and Galakatos, A. E. Radiation therapy and conservation surgery for primary and recurrent carcinoma of the vulva: Report of 40 patients and a review of the literature, Int. J. Radiat. Oncol. Biol. Phys. 14, 1123-1132 (1988).

8. Shimm, D. S., Fuller, A. F., Orlow, E. L., Dosoretz, D. E., and Aristizabal, S. A. Prognostic variables in the treatment of squamous cell carcinoma of the vulva, Gynecol. Oncol. 24, 343-358 (1986).

9. Hacker, N. F., Berek, J. S., Lagasse, L. D., Luchter, R. S., and Moore, J. G. Management of regional lymph nodes and their prognostic influence in vulvar cancer, Obstet. Gynecol. 61, 408-412 (1983).

10. Hoffman, J. S., Kumar, N. B., and Morley, G. W. Prognostic significance of groin lymph node metastases in squamous carcinoma of the vulva, Obstet. Gynecol. 66, 402-405 (1985). 